\title{
Orbital Involvement in Multiple Myeloma: A Case Report
}

\author{
Sara Terenzi, Daniela di Cristino, Alessandra Murgia, Dania Janniello, Elisabetta Ponti, \\ Barbara Tolu, Sara Cicchetti, Riccardo Santoni
}

Department of Diagnostic Imaging, Molecular Imaging, Interventional Radiology and Radiotherapy, Tor Vergata University General Hospital, Rome, Italy.

Email: sara_terenzi@fastwebnet.it

Received October $18^{\text {th }}, 2012$; revised November $19^{\text {th }}$, 2012; accepted November $30^{\text {th }}, 2012$

\begin{abstract}
Purpose: To report a retro-orbital localization of Multiple Myeloma (MM) describing its treatment and clinical result. Case Report: A 50-year-old male patient with Magnetic Resonance Imaging (MRI) evidence of a retro-orbital mass with exophthalmos, due to the pathological diagnosis of MM, was referred for Radiation Therapy (RT). Discussion: The orbital involvement in Multiple Myeloma is rare and few cases are reported in the literature. The treatment of choice is RT alone with a prescribed dose ranging between $40 \mathrm{~Gy}$ and $45 \mathrm{~Gy}$. In our patient the retro-orbital lesion, measuring $26 \times 16 \mathrm{~mm}$, was treated with Intensity Modulated Radiotherapy Technique (IMRT) delivering 4400 cGy with conventional fractionation. The treatment was well tolerated, the patient experienced a complete regression of the exophthalmos without any significant side effect.
\end{abstract}

Keywords: Plasma Cell Tumours; Multiple Myeloma; Tumours of the Orbit; Radiation Therapy

\section{Introduction}

Multiple Myeloma (MM) is a monoclonal proliferation of plasma cell that causes destructive skeletal lesions and secondary systemic effects, and is a systemic disease diagnosed in the presence of light chain (Bence Jones) proteinuria, lytic skeletal lesions, and characteristic pathology. It accounts for about $10 \%$ of the hematological malignancies, with an incidence of 5.5 cases per 100,000 inhabitants [1]. The median age at diagnosis is 70 years and only $3.4 \%$ of cases are diagnosed between 35 - 44 years of age [2]. The eye may be frequently involved but the orbital involvement is very rare [3]. In 1972, Rodman described 30 cases in the litherature, all cases presenting with some form of systemic disease [4]. More recently, Burkat et al. reported an additional 52 cases of orbital Multiple Myeloma [5]. Risk factors for orbital involvement may be related with specific immunoglobulin subtypes [6].

We report the case of a 50-year-old man who presented right exophthalmos with Magnetic Resonance (MRI) evidence of a retro-orbital mass and with a clinical and pathological history of MM.

\section{Case Report}

On August 2009 a 50-year-old male patient was referred to our Department of Radiation Oncology with a clinical history of MM diagnosed on March 2002. Since that date he underwent different treatments including allogenic bone marrow transplantation on February 2003 and irradiation of the left iliac bone (4200 cGy, 200 cGy/fraction) on summer 2007 and October 2008. On July 2009 a MRI demonstrated the presence, in the right orbital cavity, of a $26 \times 16 \mathrm{~mm}$ ovoidal-shaped lesion with regular margins which displaced laterally and superiorly the optic nerve producing exophthalmos.

On the basis of the MRI and of the clinical history, radiation treatment was advised.

The treatment was delivered using high energy photons (6 MV) produced by a Linear Accelerator Elekta Synergy ${ }^{\circledR}$ with a micro-multileaf collimator. Elekta Synergy with Beam Modulator combines a micromultileaf ( $\mu$ MLC) with a kilovolt $(\mathrm{kV})$ imaging system ca-pable of acquiring 3D X-ray volume images based on $\mathrm{kV}$ Cone Beam Computed Tomography. In order to allow precise repositioning during treatment, a personalized thermoplastic mask (Head Mask, Klarity ${ }^{\circledR}$ ) was created and fixed to a frame support (Head Frame, 3DLine ${ }^{\circledR}$ ); CT scan was obtained with a GE LightSpeed ${ }^{\circledR}$ Scanner (GE Healthcare Diagnostic Imaging, Slough, UK) with a 1.25 $\mathrm{mm}$ slice thickness and the images were exported on Syntegra software (Pinnacle, Philips Medical System, Andover, MA) to develop the IMRT treatment plan (Figure 1). During the therapy the patient's right eye glance was fixed by applying a flashing light source aligned with the immobilization system, in order to pro- 


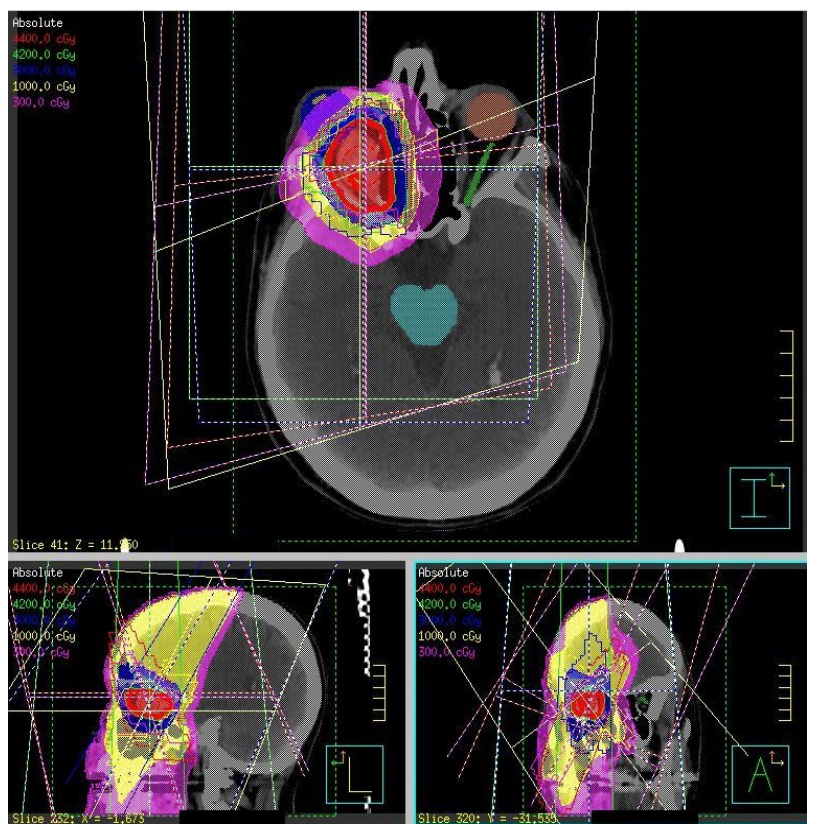

Figure 1. Axial, coronal and sagittal representation of treatment plan.

tect the ipsilateral lens. The organs at risk were the left and right eye, the left and right eye lenses, left optical nerve and the brainstem (Figure 2). Constraints for organs at risk are reported in column 2 of Table 1. All the constraints were respected as reported in column 1 where are summarized the maximum delivered doses. The treatment was started on September 9, 2009 and completed on October 12, 2009. It was well tolerated by the patient who did not complaint any significant side effect, but experiencing a complete regression of the exophthalmos and complete preservation of sight. During follow-up MRI documented a significant volume reduction of the lesion of the orbital cavity and the presence of fibrotic nodules in the right medial rectus muscle (Figure 3(a)). Two years later MRI confirmed the persistence of the fibrous residue in the right medial rectus and the absence of any local recurrence (Figure 3(b)). Exophthalmos was resolved and the patient recovered completely with preservation of visual acuity as documented by ophthalmology.

\section{Discussion}

MM is a systemic disease involving monoclonal proliferation of immune cells. Malignant plasma cell tumours are divided according to the site of origin, they can be multicenter, such as multiple myeloma, or localized, originating at bone (solitary plasmacytoma of bone) or from soft tissue (solitary extramedullary plasmacytoma). Many cases present as isolated plasmacytoma of the long bone before the diagnosis of systemic involvement. Orbital involvement as a manifestation of MM is very rare.

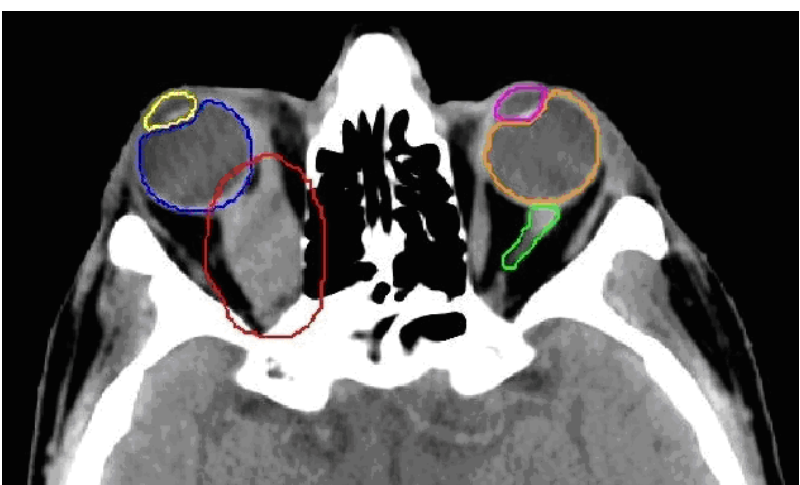

(a)

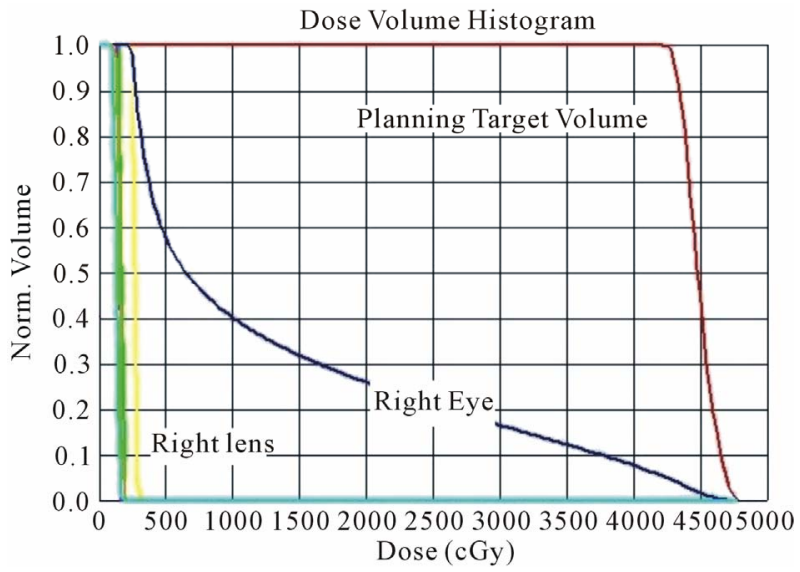

(b)

Figure 2. CT axial slice with organs at risk and planning target volume (a) and Dose Volume Histogram (b): Red is the Planning Target Volume (PTV), blue the right eye, yellow the right lens, pink the left lens, orange the left eye, light green the left optic nerve.

Table 1. Maximum delivered doses to the organs at risk (OARs).

\begin{tabular}{ccc}
\hline Organs at risk & $\begin{array}{c}\text { Maximum delivered } \\
\text { dose (cGy) }\end{array}$ & $\begin{array}{c}\text { Dose constraints } \\
\text { (cGy) }\end{array}$ \\
\hline Left eye & $\mathrm{D}_{\max } 186.7 \mathrm{cGy}$ & $\mathrm{D}_{\max }<5500 \mathrm{cGy}$ \\
Right eye & $\mathrm{D}_{\max } 4706.8 \mathrm{cGy}$ & $\mathrm{D}_{\max }<5500 \mathrm{cGy}$ \\
Left optical nerve & $\mathrm{D}_{\max } 194.1 \mathrm{cGy}$ & $\mathrm{D}_{\max }<5500 \mathrm{cGy}$ \\
Left lens & $\mathrm{D}_{\max } 160.0 \mathrm{cGy}$ & $\mathrm{D}_{\max }<1000 \mathrm{cGy}$ \\
Right lens & $\mathrm{D}_{\max } 305.6 \mathrm{cGy}$ & $\mathrm{D}_{\max }<1000 \mathrm{cGy}$ \\
Brainstem & $\mathrm{D}_{\max } 184.5 \mathrm{cGy}$ & $\mathrm{D}_{\max }<5400 \mathrm{cGy}$ \\
\hline
\end{tabular}

In our patient the ocular symptoms appeared 6 years after MM diagnosis and its first treatment. Orbitary involvement is generally unilateral and more frequently affects the sphenoid [3]. In the majority of the cases the initial symptoms are insidious with slowly progressing exophthalmos with pain, diplopia and Visual Acuity (VA) reduction [4]. Intracranial extension may lead to papille- 


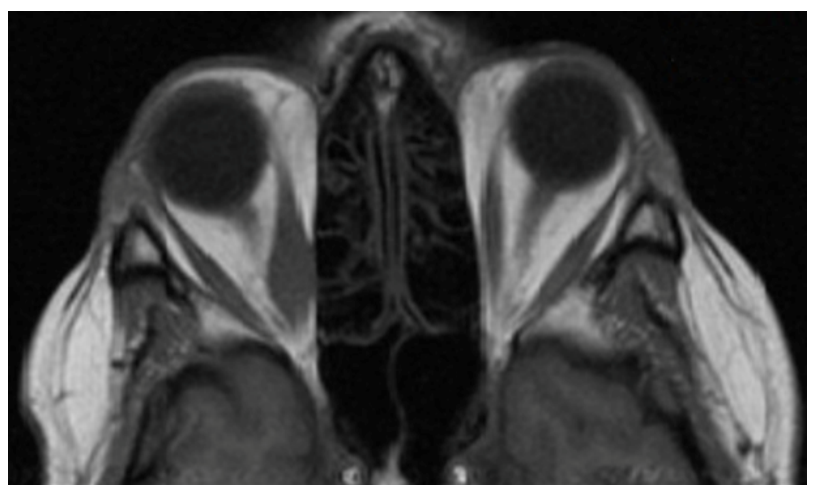

(a)

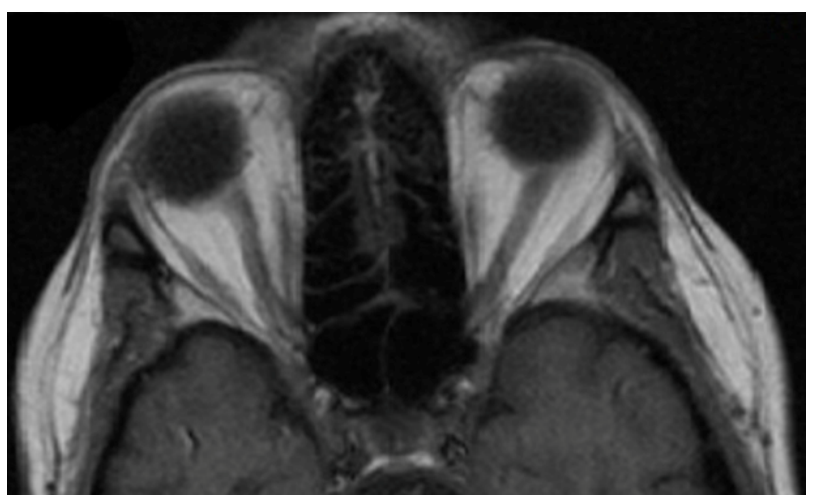

(b)

Figure 3. Six months (a) and 2 years (b) MRI since the end of radiation therapy.

dema and cranial nerve palsies. It may be accompanied by non-specific symptoms such as fever, discomfort and anorexia [7].

External beam RT is the treatment of choice in the case of localized bone or extramedullary disease [8]. The prescribed radiation dose is usually around $44 \mathrm{~Gy}$, it is delivered with conventional fractionation and it is compatible with the tolerance of most of the normal tissues surrounding the MM localization with the exception of the lens. Avoiding lens treatment to avoid a cataract following orbital irradiation is possible and feasible using a complex technique as IMRT is, and it may be used to treat even more resistant tumours involving the orbita.

This same technique was adopted with success, in our experience, in the irradiation of a patient with the diagnosis of granulocytic sarcoma of the orbital region which has been previously reported [9]. It is remarkable that both patients experienced a complete regression of the exophthalmos, any evidence of acute or late toxicity and preservation of their VA.

\section{Conclusion}

The combination of MRI and IMRT produce remarkable results in the treatment of retro-bulbar localization of different primary or metastatic tumours. IMRT allows to considerably decrease the dose to the surrounding organs at risks and in particular to the lens where detectable opacities may develop after 0.5 - 2 Gy (total dose received in a single exposure) or $5 \mathrm{~Gy}$ (total dose received in fractionated exposure).

\section{REFERENCES}

[1] M. Castro-Rebollo, R. Cañones-Zafra, E. N. VlemingPinilla, P. Drake-Rodríguez-Casanova and C. Pérez-Rico, "Unilateral Exophthalmos as the Debut of a Non-Secretory Multiple Myeloma,” Archivos de la Sociedad Española de Oftalmología, Vol. 84, No. 12, 2009, pp. 631-634. doi:10.4321/S0365-66912009001200008

[2] A. Sharma, M. Kaushal, N. K. Chaturvedi and R. Yadav, "Cytodiagnosis of Multiple Myeloma Presenting as Orbital Involvement: A Case Report,” CytoJournal, Vol. 10, 2006, pp. 3-19.

[3] A. J. Knapp, S. Gartner and P. Henkind, "Multiple Myeloma and Its Ocular Manifestations,” Survey of Ophthalmology, Vol. 31, 1987, pp. 343-351. doi:10.1016/0039-6257(87)90119-6

[4] H. L. Rodman and R. L. Font, "Orbital Involvement in Multiplemyeloma: A Review of Literature and Report of Three Cases," Archives of Ophthalmology, Vol. 87, 1972, pp. 30-35. doi:10.1001/archopht.1972.01000020032006

[5] C. N. Burkat, J. J. Van Buren and M. J. Lucarelli, “Characteristics Of Orbital Multiple Myeloma: A Case Report And Literature Review," Survey of Ophthalmology, Vol. 54, No. 6, 2009, pp. 697-704.

[6] J. Y. Chu, A. J. Lewis and D. H. Cowan, "IgDK Multiple Myeloma Presenting as Unilateral Proptosis,” Canadian Journal of Neurological Sciences, Vol. 12, No. 1, 1985, pp. 69-72.

[7] E. Clarke, "Plasma cell Myeloma of the Orbit," British Journal of Ophthalmology, Vol. 37, No. 9, 1953, pp. 543554. doi:10.1136/bjo.37.9.543

[8] P. Greenberg, R. G. Parker, Y. Fu and E. Abemayor, “The Treatment of Solitary Plasmacytoma of Bone and Extramedullaryplasmacytoma,” American Journal of Clinical Oncology, Vol. 10, No. 3, 1987, pp. 199-204. doi:10.1097/00000421-198706000-00004

[9] A. Di Marzo, G. Parca, G. Ingrosso, C. Giubilei, M. Battista, M. Benassi, et al., "Retro-Orbitalgranulocytic Sarcoma: Case Report,” Tumori, Vol. 94, No. 6, 2008, pp. 869872. 